\title{
Log-concavity of the cohomology of nilpotent Lie algebras in characteristic two
}

\author{
Grant CAIRNS \\ Department of Mathematics, La Trobe University, Melbourne, VIC 3086, Australia \\ E-mail: g.cairns@latrobe.edu.au
}

\begin{abstract}
It is known that the Betti numbers of the Heisenberg Lie algebras are unimodal over fields of characteristic two. This note observes that they are log-concave. An example is given of a nilpotent Lie algebra in characteristic two for which the Betti numbers are unimodal but not log-concave.
\end{abstract}

2000 MSC: 17B55, 17B56

The Heisenberg Lie algebra of dimension $2 m+1$ is the Lie algebra $\mathfrak{h}_{m}$ having the basis $\left\{x_{1}, \ldots, x_{m}, y_{1}, \ldots, y_{m}, z\right\}$ and nonzero relations $\left[x_{i}, y_{i}\right]=z, 1 \leq i \leq m$. For the cohomology with trivial coefficients, the Betti numbers $b_{n}=\operatorname{dim} H^{n}\left(\mathfrak{h}_{m}\right)$ have been explicitly computed in all characteristics $[1,3,4]$. Recall that the Betti numbers are unimodal if $b_{i} \leq b_{j}$ for all $0 \leq i \leq j \leq m$ and $b_{i} \geq b_{j}$ for all $m \leq i \leq j \leq 2 m+1$, and they are concave (resp., log-concave) if $b_{i}$ is at least as great as the arithmetic (resp., geometric) mean of the pair $b_{i-1}, b_{i+1}$ for all $1 \leq i \leq 2 m$. So concave implies log-concave which implies unimodal. In characteristic zero, unimodality is quite common. The Heisenberg Lie algebras play a key role in the construction of all known examples of Lie algebras in characteristic zero where the Betti numbers are not unimodal [2]. In fact, the Betti numbers of $\mathfrak{h}_{m}$ are unimodal only in characteristic two [1]. On the other hand, we know of no nilpotent Lie algebra in characteristic two whose Betti numbers fail to be unimodal. In [1], the question was posed: in characteristic two, do all nilpotent Lie algebras have unimodal Betti numbers? Since logconcavity is a common route taken to prove unimodality, it is natural to ask whether the Betti numbers of the Heisenberg algebras are unimodal in characteristic two. We record the following observation as a theorem, though it is really just a corollary of the works $[1,4]$.

Theorem 1. Over fields of characteristic two, the Betti numbers of $\mathfrak{h}_{m}$ are log-concave; i.e., $b_{n}^{2} \geq b_{n-1} b_{n+1}$ for all $n$.

Proof. For the rest of this note we fix the characteristic to be two. Emil Sköldberg showed that the Poincaré polynomial $S_{m}(t)=\sum_{n} b_{n} t^{n}$ is [4]

$$
S_{m}(t)=\frac{\left(1+t^{3}\right)(1+t)^{2 m}+\left(t+t^{2}\right)(2 t)^{m}}{1+t^{2}}
$$

Though we will not need them, we mention that the individual Betti numbers are given in [1]; for all $i \leq m$,

$$
b_{n}=\sum_{i=0}^{\left\lfloor\frac{n}{2}\right\rfloor}(-1)^{i}\left(\begin{array}{c}
2 m \\
n-2 i
\end{array}\right)+\sum_{i=0}^{\left\lfloor\frac{n-3}{2}\right\rfloor}(-1)^{i}\left(\begin{array}{c}
2 m \\
n-3-2 i
\end{array}\right)
$$

To establish the log-concavity, we observe that the Betti numbers of $\mathfrak{h}_{m+1}$ are essentially determined by those of $\mathfrak{h}_{m}$, with a curious correction for the middle two terms. Explicitly,

$$
S_{m+1}(t)=(1+t)^{2} S_{m}(t)-2^{m}\left(t^{m+1}+t^{m+2}\right)
$$


This relation is easily deduced from (1). Using induction, we assume that $S_{m}$ is log-concave. Since $(1+t)^{2}$ is log-concave, $(1+t)^{2} S_{m}(t)$ is thus also log-concave (see [5]). So in view of (2), to establish the log-concavity of $S_{m+1}$, it remains to verify it for the middle terms; that is, for $\mathfrak{h}_{m+1}$ we require that $b_{m+1}^{2} \geq b_{m} b_{m+2}$. But by Poincaré duality, $b_{m+1}=b_{m+2}$, and so we only require $b_{m+1} \geq b_{m}$, and this is given by the unimodality of the Betti numbers, which was shown in [1]. This completes the proof.

The following example shows that, despite the above result, log-concavity is not a route for establishing unimodality in the general setting of nilpotent Lie algebras in characteristic two.

Example 2. Let $\mathfrak{g}$ denote the 7-dimensional Lie algebra with basis $x_{1}, \ldots, x_{7}$ and defining relations:

$$
\begin{aligned}
& {\left[x_{1}, x_{i}\right]=x_{i+1}, \quad i=2, \ldots, 6} \\
& {\left[x_{2}, x_{i}\right]=x_{i+2}, \quad i=3,4} \\
& {\left[x_{3}, x_{4}\right]=x_{7}}
\end{aligned}
$$

Clearly $\mathfrak{g}$ is nilpotent (and actually graded and filiform). Direct calculations using Mathematica show that in characteristic two, the Betti numbers are

\begin{tabular}{c|c|c|c|c|c|c|c}
$b_{0}$ & $b_{1}$ & $b_{2}$ & $b_{3}$ & $b_{4}$ & $b_{5}$ & $b_{6}$ & $b_{7}$ \\
\hline 1 & 2 & 3 & 6 & 6 & 3 & 2 & 1
\end{tabular}

As $b_{2}^{2}<b_{1} b_{3}$, the Betti numbers are not log-concave.

\section{Acknowledgement}

The author thanks Peter Cameron for asking whether the Betti numbers of the Heisenberg Lie algebras are log-concave.

\section{References}

[1] G. Cairns and S. Jambor. The cohomology of the Heisenberg Lie algebras over fields of finite characteristic. Proc. Amer. Math. Soc., 136 (2008), 3803-3807.

[2] H. Pouseele. On the cohomology of extensions by a Heisenberg Lie algebra. Bull. Austral. Math. Soc., 71 (2005), 459-470.

[3] L. J. Santharoubane. Cohomology of Heisenberg Lie algebras. Proc. Amer. Math. Soc., 87 (1983), 23-28.

[4] E. Sköldberg. The homology of Heisenberg Lie algebras over fields of characteristic two. Math. Proc. R. Ir. Acad., 105A (2005), 47-49 (electronic).

[5] R. P. Stanley. Log-concave and unimodal sequences in algebra, combinatorics, and geometry. In "Graph Theory and Its Applications: East and West" (Jinan, 1986). M. F. Capobianco, M. G. Guan, D. F. Hsu, and F. Tian, Eds. Annals of the New York Academy of Sciences 576, New York Academy of Sciences, New York, 1989, 500-535.

Received April 18, 2009

Revised July 24, 2009 